\title{
Cognitive Behavioral Therapy for Sleep in Cancer Patients: Research, Techniques, and Individual Considerations
}

\author{
LAURA MELTON, PhD, ABPP
}

From University of Colorado Denver, Anschutz Medical Campus, Denver, Colorado Author's disclosures of conflicts of interest are found at the end of this article.

Correspondence to: Laura Melton, PhD, ABPP, MS 8117, 12801 E 17th Ave, Aurora, C0 80045. E-mail: laura.melton@ucdenver.edu https://doi.org/10.6004/jadpro.2018.9.7.4 (c) 2018 Harborside $^{\mathrm{TM}}$
J Adv Pract Oncol 2018:9(7):732-740

\begin{abstract}
This article reviews cognitive behavioral therapy techniques for sleep in the cancer realm. There are unique factors associated with cancer that can exacerbate sleep issues. Stimulus control, sleep restriction, relaxation, paradoxical intention, and cognitive therapy are discussed as specific components of cognitive behavioral therapy for sleep, and practical techniques for implementation are provided. Individual considerations in implementing these techniques are also discussed, including factors such as age, ethnicity, gender, relationship status, educational level, work status, cancer history, pharmacologic treatments, and health beliefs.
\end{abstract}

leep is a necessary component of life; humans spend about one-third of their lives asleep (Dresler et al., 2014). Sleep helps restore the body, plays a role in temperature regulation, and influences the immune system and hormones (Hearson \& Sawatzky, 2008; Van Someren et al., 2015). Sleep drive and cycles are affected by the circadian rhythm, the body's clock that is controlled by the hypothalamus and influenced by external cues such as activities, light, social interactions, and food, as well as by sleep debt and sleep drive, which are based on prior amounts of sleep and wakefulness (Berger, 2009; Dresler et al., 2014; Van Someren et al., 2015).

People struggle with sleep for various reasons, and there are many different types of sleep problems. In fact, sleep researchers have defined over 100 different sleep disorders. With insomnia, some people have difficulties falling asleep or staying asleep, some do not get restorative sleep, and others have a combination of these difficulties (Berger, 2009). Research suggests that even after the primary cause of insomnia is resolved, many individuals continue to 
experience sleep issues as a result of conditioning and other behavioral and cognitive factors (Buysse, 2003).

According to a report by the Institute of Medicine Committee on Sleep Medicine and Research (Institute of Medicine, 2006), 50 to 70 million Americans suffer from sleep disorders and sleep deprivation. This societal issue with sleep has staggering consequences: Almost 20\% of serious car crash injuries and $30 \%$ of heavy trucking accidents that were fatal to the driver were associated with sleeping at the wheel or driver sleepiness (The Royal Society for the Prevention of Accidents, 2001). Also, fatigue was considered a factor in impairing judgment prior to the NASA space shuttle Challenger explosion, Chernobyl, and the Three Mile Island accident, as well as the Exxon Valdez oil spill (Center for Homeland Defense and Security, 1989; National Aeronautics and Space Administration, 1979, 1986; United States Nuclear Regulatory Commission, 2016).

\section{SLEEP |N CANCER}

Insomnia and other sleep-wake disturbances are twice as high in individuals with cancer as compared to the general population (National Cancer Institute, 2010). Cancer-related insomnia can exist for years after cancer treatment ends (Garland et al., 2014; Kotronoulas, Wengström, \& Kearney, 2012). Insomnia in cancer patients has been related to pain and depression, decreased quality of life, impaired immune function, cognitive and functional issues, poorer overall survival, and even increased risk of death (Berger, 2009; Chen, Yu, \& Yang, 2008; Fiorentino \& Ancoli-Israel, 2007; Hoffman, Given, von Eye, Gift, \& Given, 2007; McMillan, Tofthagen, \& Morgan, 2008).

Individuals with cancer may be susceptible to developing insomnia based on recommendations by health-care staff to rest, nap, and decrease normal activity levels (Savard \& Morin, 2001). Although insomnia is beginning to gain recognition in the cancer field, it is still underassessed and undertreated. Fortunately, once assessed and diagnosed, there are many treatments for insomnia. Although many therapies exist to treat insomnia (yoga, massage, guided imagery, education, exercise, etc.), cognitive behavioral therapy (CBT) and the individualized components are the only therapeutic techniques considered effective in the treatment of insomnia (Morin et al., 2006).

Despite many people using pharmacotherapy to treat insomnia (Savard \& Morin, 2001), there is no reliable evidence that shows effectiveness in using pharmacotherapy for the treatment of sleep in the cancer patient population (Howell et al., 2013), and experts report CBT for insomnia (CBTI) to be the best first-line treatment for insomnia in this population (Savard, 2005). The American Academy of Sleep Medicine has called CBT-I "the treatment of choice for insomnia" (Harvey, 2010). As a result of the research on CBT in cancer patients with insomnia, CBT-I is in the most highly recommended category of intervention ("Recommended for Practice") by the 2014 Oncology Nursing Society Putting Evidence into Practice resource (Irwin \& Johnson, 2014).

\section{WHAT IS COGNITIVE BEHAVIORAL THERAPY FOR INSOMNIA?}

Cognitive behavioral therapy is an excellent treatment choice for helping with insomnia. As the name of the therapy suggests, CBT focuses on how behavior and cognition (beliefs, thoughts, etc.) affect sleep. Cognitive behavioral therapy for insomnia can help with potential behavioral difficulties by first identifying them and then changing sleeprelated behaviors that are incompatible with good sleep. Some of these behavioral changes include changes that retrain circadian rhythms and reduce arousal or stimulation when trying to go to sleep.

The cognitive changes in CBT for sleep problems include, based on an individual's sleep needs, changing thoughts and beliefs about sleep that may be interfering with good sleep. Cognitive behavioral therapy can also help set appropriate expectations for sleep. The expectation of being able to sleep for 9 hours straight every night, to fall asleep every night within 5 minutes, and to wake up feeling refreshed without having to get up to go to the bathroom is probably unrealistic. The cognitive portion of CBT can also help with thoughts and beliefs that may be increasing arousal.

Cognitive behavioral therapy for insomnia is considered to be one of the most effective treatments for insomnia, and some of its components are also independently regarded as effective 
treatments for insomnia. There are currently four different CBT-based techniques that have been found to be helpful for insomnia and sleep issues (Edinger \& Means, 2005; Howell et al., 2013; Morin et al., 2006), and independently of CBT meet the standards for empirically supported treatments (Chambless et al., 1998). These four CBT-I components are stimulus control therapy, sleep restriction therapy, relaxation, and paradoxical intention.

\section{Stimulus Control}

Difficulty sleeping can come in many different forms. Stimulus control interventions are aimed at helping people reconnect with bedtime. They are positive, sleep inducing, and identify and diminish behaviors that may be hindering sleep. These behavioral suggestions also help people reestablish a regular sleep-wake cycle with a 24 -hour circadian rhythm. Making a better environment to sleep in, associating the bed with sleep, and reconditioning the body when and where to sleep fall under the categories of stimulus control and sleep restriction.

Stimulus control is based on the idea that insomnia or sleep issues are the result of conditioning the bed and bedroom with activities other than sleep (Ebben \& Spielman, 2009; Edinger \& Means, 2005; Schutte-Rodin, Broch, Buysse, Dorsey, \& Sateia, 2008; Woodward, 2011). Stimulus control works to create a more appropriate sleep-wake cycle and reassociate the bed with falling asleep quickly. Table 1 lists specific techniques used in stimulus control for insomnia (included are some sleep and hygiene suggestions that overlap greatly with stimulus control and reconditioning the bed and nighttime with sleep).

Stimulus control techniques are unlikely to work well if they are not exercised consistently. Individuals should plan to spend at least 1 to 2 weeks on each technique before noticing a change in sleep, as unconditioning and reconditioning take time.

\section{Sleep Restriction}

Sleep restriction was developed in the 1980 s based on the idea that excess time in bed perpetuates insomnia, and subsequently restricting sleep time strengthens the association between sleep and bed, and increases sleep drive (Ebben \& Spielman, 2009; Edinger \& Means, 2005;
Schutte-Rodin et al., 2008; Woodward, 2011). Sleep restriction helps consolidate sleep, making the time used for sleeping more efficient, with the aim of sleep efficiency being $85 \%$ to $90 \%$. Sleep efficiency can be calculated with the following equation:

Total sleep time $\div$ Total time in bed $\times 100=$ Sleep efficiency (\%)

For example, if an individual were to go to bed at 10:30 pm and get up at 6:30 am, they would have spent a total of 8 hours in bed. However, if that individual did not fall asleep until 11:30 pm and was awake for 1 hour in the middle of the night, the total sleep time would be 6 hours. Thus, that individual's sleep efficiency would be:

Total sleep time (6 hours) $\div$ Total time in bed ( 8 hours $) \times 100=75 \%$ sleep efficiency

With a sleep efficiency goal of $85 \%$ to $90 \%$ (there are variations in the literature about what an ideal sleep efficiency goal is; some say $80 \%$ to $90 \%$, others say $85 \%$ or higher), behavioral sleep restriction involves decreasing the amount of time an individual is in bed to increase sleep efficiency. Most people do not like this at first because it seems paradoxical; they question why they should spend less time in bed when their goal is more sleep. However, by spending excess time in bed trying to force or will oneself to sleep, that individual can actually have reduced sleep quality, sleep fragmentation, and lighter sleep. The goal of sleep restriction is to consolidate sleep so that sleep is less fragmented.

Under sleep restriction, most individuals coming for CBT-I will spend less time in bed. Depending on their sleep efficiency, they will set a later bedtime and an established wake-up time. They will not go to bed until their new bedtime, even if they think they can go to sleep earlier. Importantly, in the morning, no matter how tired they are or how tempted they are to linger in bed, individuals will get up at their new wake-up time. Completing these steps will increase the likelihood of being sleepy enough to fall asleep more easily the next night. The steps for sleep restriction are as follows.

Step 1. Determine baseline average total sleep time and time in bed. Use a sleep diary to track sleep for 1 to 2 weeks.

Step 2. Change total time in bed to match total sleep time, keeping the wake-up time fixed. 


\section{Table 1. Techniques Used in Stimulus Control for Insomnia}

\section{Technique}

1. The bed is for sleep and sex only. No other activities should occur in the bed.

2. Avoid daytime napping.

3. Eliminate visual and auditory distractions at night.

4. Create a bedtime ritual that allows relaxation to start before bedtime.

5. Go to bed and wake up at the same time each day.

6. Do not spend more than 20 minutes awake in bed.

7. Do not engage in arousing activities before bed or in the middle of the night. Some people benefit from stopping caffeine consumption after noon. Avoid alcohol at night. Avoid spicy or arousing food before bed.

8. Upon waking up, eat something. Get sunlight or artificial light in the morning.

\section{Notes}

This works to reestablish the bed as a place of sleep and sex, and nothing else.

Daytime napping can reduce the drive for sleep at nighttime. During the day, sleep debt is built; if there is not enough sleep debt by the end of the day, it can be difficult to fall asleep. Napping can take away part of the sleep debt; activities such as being awake, exercising, and being engaged in activities create more sleep debt. By staying awake during the day there is a higher likelihood that the individual will be more tired at night, thus helping to reestablish the sleep-wake cycle.

Sleeping in a dark, quiet, slightly cool room may make it easier to fal asleep. A white-noise machine may be beneficial if the room feels too quiet. This technique helps eliminate any information that may distract one from falling asleep. In today's technological age, this may include blocking out light from various gadgets, including cell phones, computers, and other plugged-in devices. Some people also benefit from blackout curtains and turning alarm clocks backwards if they emit light.

Warm showers or baths, warm beverages, soft music, and relaxing reading are all ways to help ease the transition from alert to asleep. They can also provide cues to the body that sleep lies ahead and may help condition the body to be prepared for sleep.

This will help condition the body to know when to expect sleep vs. when to expect arousal. Many people have the tendency to want to sleep in on days they are not working, but this can disrupt the sleepwake cycle, similar to jet lag.

Spending more than 20 minutes awake in bed may recondition the bed with not sleeping. If not asleep after 20 minutes, get out of bed and engage in a neutral activity. Avoid arousing activities such as television, computer, or eating because those activities may become associated with rewards for not sleeping. Return to bed when sleepy. Repeat if after returning to bed one is still not asleep after 20 minutes. Avoid watching the clock; instead, leave the room after 20 minutes.

Although alcohol helps some people become sleepy or fall asleep, it can disrupt nighttime sleep. Avoiding spicy or arousing food decreases the likelihood of having acid reflux or digestive issues, which may contribute to sleep difficulties.

These activities can reinforce or train the body about the correct sleep-wake cycle.
Step 3. Calculate sleep efficiency: Total sleep time $\div$ Total time in bed $\times 100=$ Sleep efficiency $(\%)$

Table 2 lists recommendations for adjusting time in bed based on sleep efficiency scores.

\section{Relaxation}

Relaxation techniques can help with both cognitive and physiological arousal that can cause sleep difficulties in some people (Ebben \& Spielman, 2009; Edinger \& Means, 2005; Schutte-Rodin et al., 2008; Woodward, 2011). Individuals can be trained in various types of relaxation techniques such as guided imagery, diaphragmatic breathing, progressive muscle relaxation, and passive progressive muscle relaxation. Patients are asked to practice these techniques at home, and once mastered can help promote a restful state to help the individual relax and fall asleep.

From a physiological standpoint, relaxation can be helpful in reducing arousal within the body. The "fight-or-flight response" refers to the physiological reaction that occurs in the body in the face of a perceived threat. For example, if an individual were in the wilderness and a lion ap- 


\section{Table 2. Recommendations for Individuals Based on Sleep Efficiency Score \\ Sleep efficiency score Recommendation \\ $\geq 91 \%$ \\ $85 \%-90 \%$ \\ $<84 \%$ \\ The individual might not be spending enough time in bed, and this may be a sign that the body is actually craving more sleep. Increase time in bed by 15 to 30 minutes. Reassess every week and make adjustments as needed. Repeat until sleep efficiency score is consistently in the $85 \%-90 \%$ range. Do not nap, as this fragments sleep. \\ Stay with current sleep schedule and reassess after 1 week. Make adjustments as needed. \\ The individual is spending too much time in bed based on how much they are actually sleeping. Decrease time in bed by 15 to 30 minutes. Reassess every week and make adjustments as needed. Repeat until sleep efficiency score is consistently in the $85 \%-90 \%$ range. Do not nap, as this fragments sleep.}

proached, he/she would do one of three things: fight the lion, flee from the lion, or play dead. The human body has a built-in reaction to help itself have energy to fight or flee when faced with the lion. However, in today's society, individuals are rarely faced with lions, and yet the body has the same physiological response when individuals feel threatened. Individuals can have the same energy reaction in their bodies when they feel threatened by their boss, another driver, or problems sleeping. In a predominantly physiologically safe environment, individuals do not need as much energy to deal with sleep problems, yet bodies have not evolved to respond to the new types of threat, which do not require the ability to fight or flee in many stress-inducing situations.

Some individuals experience arousal issues that make it difficult to sleep. The system in the human body that produces the fight-or-flight response is the sympathetic nervous system. The sympathetic nervous system makes sure that in times of potential danger, the body has sufficient energy to fight or flee, the blood supply is centered in the middle of the body where vital organs are centered, and the body is not engaged in nonessential behaviors such as bowel and bladder function.

The companion to the sympathetic nervous system is the parasympathetic nervous system. The parasympathetic nervous system acts like a parachute and helps to slow down the body's reaction, ultimately bringing it back to its normal state of homeostasis. Thus, after being aroused, the body eventually returns back to its normal state by having the sympathetic nervous system reduce itself and having the parasympathetic nervous system increase. Relaxation techniques, once mastered, can be used to return the body to homeostasis by activating the parasympathetic nervous system and training it to help reduce the reaction to stress.

\section{Paradoxical Intention}

Many insomnia sufferers have increased sleep issues because they worry about not being able to sleep. Paradoxical intention reduces such anticipatory anxiety by confronting the fear of staying awake (Ebben \& Spielman, 2009; Edinger \& Means, 2005; Schutte-Rodin et al., 2008; Woodward, 2011). Individuals are told to stay awake as long as possible, and because they have been given permission to not sleep, they should have decreased anxiety and feel more relaxed and then fall asleep more quickly.

\section{COGNITIVE THERAPY}

Many individuals with sleep issues have unhelpful or dysfunctional thoughts about sleep, which cause them more sleep difficulty (Ebben \& Spielman, 2009; Edinger \& Means, 2005; Schutte-Rodin et al., 2008; Woodward, 2011). Cognitive restructuring techniques teach individuals to identify, evaluate, and challenge their thoughts about sleep. Put simply, the four steps in cognitive restructuring include: (1) Identifying negative or irrational thoughts or ideas; (2) Identifying the thinking errors involved in negative or irrational thoughts or ideas; (3) Disputing and challenging negative or irrational thoughts or ideas; and (4) Creating an alternative, rational thought for the future based on the actual evidence.

Common insomnia-related thinking errors include misconceptions about what caused an individual's insomnia (e.g., "A chemical imbalance caused my insomnia"), catastrophizing and 
overestimating thoughts about the consequences of insomnia (e.g., "If I don't get enough sleep tonight I will fail my test tomorrow"), thoughts about sleep that may be true but are unhelpful (e.g., "I'm having a hard time getting to sleep"), unrealistic expectations about sleep (e.g., "I need 10 hours of sleep each night without getting up to go to the bathroom"), and incorrect beliefs about how to get to sleep (e.g., "I should be able to force myself to sleep right now"). Many individuals also experience anxious thoughts that are unrelated to their insomnia but that cause them to have difficulty sleeping, such as finances, family, work, health, etc.

Together, these techniques (cognitive therapy, stimulus control, sleep restriction, relaxation training, and paradoxical intention) have strong empirical support for the treatment of insomnia. Cognitive behavioral therapy for insomnia has also been found to be effective for individuals with cancer (Berger, 2009; Irwin \& Johnson, 2014), who tend to have an increased prevalence of insomnia (National Cancer Institute, 2010).

\section{INDIVIDUAL CONSIDERATIONS IN IMPLEMENTATION}

There are unique individual factors that are important to assess, consider, and incorporate into CBT sleep work. A nonexhaustive list of individual factors that are important to consider in CBT sleep work are presented here.

\section{Age}

Research suggests that sleep needs change over the lifetime (Mukherjee et al., 2015). Infants need the most sleep, typically 12 to 18 hours each day. By the teenage years, sleep needs have dropped to 8.50 to 9.25 hours each day. Adults vary widely, from some needing 10 hours each day to others needing just 5 hours each day, but most need 7 or more hours each day. The average American adult gets 6.90 hours of sleep during the week and 7.50 hours of sleep during the weekend; however, 39\% of those Americans polled reported sleeping less than 7 hours on weeknights (National Sleep Foundation, 2002). It is important to be cognizant as to whether there is true insomnia, or whether an individual's sleep needs have changed over time (Mukherjee et al., 2015).

\section{Ethnicity}

Some research suggests that Asians are less likely to use pharmacological sleep aids and more likely to rely on research and recommendations on techniques to help them sleep (National Sleep Foundation, 2010). However, compared to Whites (56\%), Blacks (45\%), and Hispanics $(48 \%)$, Asians (31\%) are the least likely to discuss with or have been asked about sleep by their doctors or health-care providers (National Sleep Foundation, 2010). Additionally, Asians (10\%) are half as likely to be diagnosed with a sleep disorder (sleep apnea, insomnia, restless leg syndrome, circadian rhythm disorder, narcolepsy, and other) as compared with Whites (20\%), Blacks, (19\%) and Hispanics (19\%; National Sleep Foundation, 2010). What remains unclear is whether Asians truly have a lower occurrence of sleep issues or are underassessed and subsequently underdiagnosed. Also of note, unlike Whites, Blacks, or Hispanics, research suggests that Asians are just as likely to report using "sleep aids" regardless of whether they reported getting a good night's sleep every night or almost every night (24\%) or less often (26\%; National Sleep Foundation, 2010).

\section{Gender}

Research has found that despite having higher sleep-related complaints than men, women actually have higher sleep quality when compared to men (Krishnan \& Collop, 2006). Hormone-driven occurrences, such as puberty, menstruation, pregnancy, and menopause, may increase the gender gap in sleep complaints with age, as sleep architecture can be affected by hormone-driven changes (Krishnan \& Collop, 2006).

\section{Relationship Status}

Research suggests that widowed individuals are at an increased risk for developing insomnia (Monk, Germain, \& Reynolds, 2008).

\section{Educational Level}

An individual's educational level is important to know in order to help gauge an appropriate level for psycho-education and intervention. Educational level is also associated with insomnia (Tjepkema, 2005). 


\section{Work Status}

Unemployed individuals are at an increased risk for developing insomnia (Tjepkema, 2005).

\section{Cancer History}

Precipitating factors for insomnia in patients with cancer include the distress, worry, and anxiety that can accompany a diagnosis of cancer, as well as pain, side effects of treatment (e.g., fatigue, nausea, vomiting, etc.), and/or the direct iatrogenic effects of treatment on sleep (Roscoe et al., 2007). Correlations have been found between sleep and chemotherapy, radiation therapy, and surgery for a wide range of cancer diagnoses (Roscoe et al., 2007). Adjunct hormone therapies, such as aromatase inhibitors, are now considered standard treatments to decrease the risk of recurrence in breast cancer patients. However, over $50 \%$ of women on aromatase inhibitors complain of insomnia (Desai et al., 2012).

\section{Pharmacologic Treatments}

At one point, approximately $25 \%$ to $50 \%$ of all prescriptions written for cancer patients were for sleep aids (Derogatis et al., 1979; Stiefel, Kornblith, \& Holland, 1990). More recent research suggests that in women with breast cancer, $46 \%$ received a prescription sedative or hypnotic to help with sleep, while $24 \%$ used an over-the-counter analgesic for sleep (Moore, Berger, \& Dizona, 2011). When comparing CBT with sleep medications for insomnia, the research suggests that CBT has longer-term effectiveness, while sleep medications are more helpful for the short term (Jacobs, PaceSchott, Stickgold, \& Otto, 2004).

\section{Health Beliefs}

There is a lack of evidence substantiating stress and insomnia as causes of breast cancer or cancer recurrence, yet in research, $42 \%$ of women attribute the cause of their breast cancer to stress, and $28 \%$ attribute their lack of recurrence to stress reduction, despite this lack of evidence (Stewart et al., 2001).

\section{CONCLUSIONS AND CLINICAL IMPLICATIONS}

Insomnia and sleep issues are prevalent in the cancer realm. The occurrence of insomnia in pa- tients with cancer is frequent and often severe enough to warrant intervention. In planning supportive care services for cancer patients, prevention and early detection could be helpful to the patient, improve patient outcomes, and prevent suffering and larger medical costs in the future. Universal screening about sleep issues at every health-care encounter can elevate the conversation and let patients know it is appropriate to talk about sleep with any provider given the far-reaching effects of sleep issues. Small, quick interventions for sleep, which could easily be delivered in a health-care setting, could reduce suffering and even save lives. Sleep interventions do not have to be long and complicated, but can be adapted to fit the clinical setting and lifestyle of the individual while still providing positive outcomes (Woodward, 2011).

\section{Disclosure}

The author has no conflicts of interest to disclose.

\section{References}

Berger, A. M. (2009). Update on the state of the science: Sleep-wake disturbances in adult patients with cancer. Oncology Nursing Forum, 36(4), E165-E177. https:/doi. org/10.1188/09.ONF.E165-E177

Buysse, D. J. (2003). Diagnosis and classification of insomnia disorders. In M. P. Szuba, J. D. Kloss, \& D. F. Dingers (Eds.), Insomnia: Principles and management (pp. 3-22). Cambridge, England: Cambridge University Press.

Center for Homeland Defense and Security. (1989). Marine accident report: Grounding of the U.S. Tankship Exxon Valdez on Bligh Reef, Prince William Sound Near Valdez, Alaska. Retrieved from https://www.hsdl. org/?abstract\&did=746707

Chambless, D. L., Baker, M. J., Baucom, D. H., Beutler, L. E., Calhoun, D. S., Crits-Christoph, P.,...Woody, S. R. (1998). Update on empirically validated therapies, II. The Clinical Psychologist, 51(1), 3-16.

Chen, M. L., Yu, C. T., \& Yang, C. H. (2008). Sleep disturbances and quality of life in lung cancer patients undergoing chemotherapy. Lung Cancer, 62(3), 391-400. https://doi. org/10.1016/j.lungcan.2008.03.016

Derogatis, L. R., Feldstein, M., Melisaratos, N., Morrow, G., Schmale, A., Schmitt, M.,...Adler, L. M. (1979). A survey of psychotropic drug prescriptions in an oncology population. Cancer, 44(5), 1919-1929. https://doi. org/10.1002/1097-0142(197911)44:5\%3C1919::AID CNCR2820440555\%3E3.0.CO;2-T

Desai, K., Mao, J. J., Su, I., DeMichele, S., Li, Q., Xie, S. X., \& Gehrman, P. R. (2012). Prevalence and risk factors for insomnia among breast cancer patients on aromatase inhibitors. Supportive Care in Cancer, 21(1), 43-51. https:// doi.org/10.1007/s00520-012-1490-z

Dresler, M., Spoormaker, V. I., Beitinger, P., Czisch, M., Kimu- 
ra, M., Steiger, A., \& Holsboer, F. (2014). Neurosciencedriven discovery and development of sleep therapeutics. Pharmacology and Therapeutics, 141(3), 300-334. https:// doi.org/10.1016/j.pharmthera.2013.10.012

Ebben, M. R., \& Spielman, A. J. (2009). Non-pharmacological treatment for insomnia. Journal of Behavioral Medicine, 32(3), 244-254. https://doi.org/10.1007/s10865-008-9198-8

Edinger, J. D., \& Means, M. K. (2005). Cognitive-behavioral therapy for primary insomnia. Clinical Psychology Review, 25(5), 539-558. https://doi.org/10.1016/j.cpr.2005.04.003

Fiorentino, L., \& Ancoli-Israel, S. (2007). Sleep dysfunction in patients with cancer. Current Treatment Options in Neurology, 9(5), 337-346. https://doi.org/10.1007/s11940007-0019-0

Garland, S. N., Johnson, J. A., Savard, J., Gehrman, P., Perlis, M., Carlson, L., \& Campbell, T. (2014). Sleeping well with cancer: A systematic review of cognitive behavioral therapy for insomnia in cancer patients. Neuropsychiatric Disease and Treatment, 10, 1113-1124. https://doi.org/10.2147/ NDT.S47790

Harvey, A. G. (2010). Insomnia. In D. McKay, J. S. Abramowitz, \& S. Taylor (Eds.), Cognitive- behavioral therapy for refractory cases: Turning failure into success (pp. 277-295). Washington, DC: American Psychological Association. https://doi.org/10.1037/12070-013

Hearson, B., \& Sawatzky, J. V. (2008). Sleep disturbances in patients with advanced cancer. International Journal of Palliative Nursing, 14(1), 30-37. https://doi.org/10.12968/ ijpn.2008.14.1.28150

Hoffman, A. J., Given, B. A., von Eye, A., Gift, A. G., \& Given, C. W. (2007). Relationships among pain, fatigue, insomnia and gender in persons with lung cancer. Oncology Nursing Forum, 34(4), 785-792. https://doi.org/10.1188/07.ONF.785-792

Howell, D., Oliver, T. K., Keller-Olaman, S., Davidson, J. R., Garland, S., Samuels, C.,...Taylor, C. (2013). Sleep disturbance in adults with cancer: A systematic review of evidence for best practices in assessment and management for clinical practice. Annals of Oncology, 25(4), 791-800. https://doi. org/10.1093/annonc/mdt506

Institute of Medicine. (2006). Sleep disorders and sleep deprivation: An unmet public health problem. Washington, DC: The National Academies Press.

Irwin, M., \& Johnson, L. (2014). Putting evidence into practice. Pittsburgh, Pennsylvania: Oncology Nursing Society.

Jacobs, G. D., Pace-Schott, E. F., Stickgold, R., \& Otto, M. W. (2004). Cognitive behavioral therapy and pharmacotherapy for insomnia: A randomized controlled trial and direct comparison. Archives of Internal Medicine, 164(17), 18881896. https://doi.org/10.1001/archinte.164.17.1888

Kotronoulas, G., Wengström, Y., \& Kearney, N. (2012). A critical review of women's sleep-wake patterns in the context of neo-/adjuvant chemotherapy for early-stage breast cancer. Breast, 21(2), 128-141. https://doi.org/10.1016/j. breast.2011.12.004

Krishnan, V., \& Collop, N. A. (2006). Gender differences in sleep disorders. Current Opinion in Pulmonary Medicine, 12(6), 383-389. https://doi.org/10.1097/01. mcp.0000245705.69440.6a

McMillan, S. C., Tofthagen, C., \& Morgan, M. A. (2008). Relationships among pain, sleep disturbances, and depressive symptoms in outpatients from a comprehensive cancer center. Oncology Nursing Forum, 35(4), 603-611. https://doi. org/10.1188/08.ONF.603-611
Monk, T. H., Germain, A., \& Reynolds, C. F. (2008). Sleep disturbance in bereavement. Psychiatric Annals, 38(10), 671-675. https://doi.org/10.3928/00485713-20081001-06

Moore, T. A., Berger, A. M., \& Dizona, P. (2011). Sleep aid use during and following breast cancer adjuvant chemotherapy. Psycho-Oncology, 20(3), 321-325. https://doi.org/10.1002/ pon.1756

Morin, C. M., Bootzin, R. R., Buysse, D. J., Edinger, J. D., Espie, C. A., \& Lichstein, K. (2006). Psychological and behavioral treatment of insomnia: Update of the recent evidence (1998-2004). Sleep, 29(11), 1398-1414. https://doi. org/10.1093/sleep/29.11.1398

Mukherjee, S., Patel, S. R., Kales, S. N., Ayas, N. T., Strohl, K. P., Gozal, D., \& Malhotra, A. (2015). An official American Thoracic Society statement: The importance of healthy sleep. Recommendations and future priorities. American Journal of Respiratory and Critical Care Medicine, 191(12), 14501458. https://doi.org/10.1164/rccm.201504-0767ST

National Aeronautics and Space Administration. (1986). Report of the Presidential Commission on the space shuttle challenger accident. Retrieved from https://history.nasa.gov/ rogersrep/v2appg.htm

National Cancer Institute. (2010). Sleep disorders (PDQ). Retrieved from https:/www.cancer.gov/about-cancer/treatment/side-effects/sleep-disorders-pdq

National Sleep Foundation. (2002). Sleep in America poll. Retrieved from http://www.sleepfoundation.org/sites/default/files/2002SleepInAmericaPoll.pdf

National Sleep Foundation. (2010). Sleep in America poll. Sleep and Ethnicity. Retrieved from http://www.sleepfoundation. org/article/sleep-america-polls/2010-sleep-and-ethnicity

Roscoe, J. A., Kaufman, M. E., Matteson-Rusby, S. E., Palesh, O. G., Ryan, J. L., Kohli, S.,...Morrow, G. R. (2007). Cancerrelated fatigue and sleep disorders. Oncologist, 12(suppl 1), 35-42. https://doi.org/10.1634/theoncologist.12-S1-35

Savard, J. (2005). Are refined differential diagnosis and pharmacological treatment for cancer-related insomnia always necessary? Practical recommendations for cancer care providers. Supportive Oncology, 3(5), 361-363.

Savard, J., \& Morin, C. M. (2001). Insomnia in the context of cancer: A review of a neglected problem. Journal of Clinical Oncology, 19(3), 895-908. https://doi.org/10.1200/ JCO.2001.19.3.895

Schutte-Rodin, S., Broch, L., Buysse, D., Dorsey, C., \& Sateia, M. (2008). Clinical guidelines for the evaluation and management of chronic insomnia in adults. Journal of Clinical Sleep Medicine, 4(5), 487-504.

Stewart, D. E., Cheung, A. M., Duff, S., Wong, F., McQuestion, M., Cheng, T.,...Bunston, T. (2001). Attributions of cause and recurrence in long-term breast cancer survivors. Psycho- Oncology, 10(2), 179-183. https://doi.org/10.1002/pon.497

Stiefel, F. C., Kornblith, A. B., \& Holland, J. C. (1990). Changes in the prescription patterns of psychotropic drugs for cancer patients during a 10-year period. Cancer, 65(4), 1048-1053. https://doi. org/10.1002/1097-0142(19900215)65:4\%3C1048::AIDCNCR2820650434\%3E3.0.CO;2-N

The Royal Society for the Prevention of Accidents. (2001). Driver fatigue and road accidents: A literature review and position paper. Retrieved from http://www.rospa.com/rospaweb/docs/advice-services/road-safety/drivers/fatiguelitreview.pdf

Tjepkema, M. (2005). Insomnia. Health Reports, 17(1), 9-25 
United States Nuclear Regulatory Commission. (1987). Report on the accident at the Chernobyl nuclear power station (NUREG-1250). Retrieved from https://www.nrc.gov/ reading-rm/doc-collections/nuregs/staff/sr1250/

United States Nuclear Regulatory Commission. (1979). Investigation into the March 28, 1979 Three Mile Island Accident by Office of Inspection and Enforcement. Retrieved from https://tmi2kml.inl.gov/Documents/2c-L2-NUREG/ NUREG-0600,\%20Investigation\%20into\%20the\%20 03-28-1979\%20TMI\%20Accident\%20by\%20the\%200f-
fice\%20of\%20IE\%20(1979-08).pdf

Van Someren, E. J. W., Cirelli, C., Dijk, D. J., Van Cauter, E., Schwartz, S., \& Chee, M. W. L. (2015). Disrupted sleep: From molecules to cognition. Journal of Neuroscience, 35(41), 13889-13895. https://doi.org/10.1523/JNEUROSCI.2592-15.2015

Woodward, S. C. (2011). Cognitive-behavioral therapy for insomnia in patients with cancer. Clinical Journal of Oncology Nursing, 15(4), E42-E51. https://doi.org/10.1188/11.CJON. E42-E52 